\title{
Vom Blitzableiter zur Bad-Bank
}

\author{
Die Debatten um die Treuhandanstalt - und was sich \\ daraus über das Verhältnis von Politikwissenschaft und \\ Zeitgeschichtsforschung lernen lässt
}

\section{Marcus Böick}

Online publiziert: 4. August 2020

(C) Der/die Autor(en) 2020

\section{Einleitung: der Schock von 1990 und die Starre von 2020}

Löst die „Neue Normalität“ des Jahres 2020 nun endgültig das vielzitierte (und meist wenig gelesene) „Ende der Geschichte“ des Jahres 1990 als zentrale epochale Zäsur $\mathrm{ab}$ ? Wie verhalten sich andere historische Einschnitte der vergangenen Jahrzehnte - etwa die Jahre 2001 (Terrorattacken), 2008 (Finanzmarktkrisen) oder 2015 (Migrationsdebatten) - zu diesen allerjüngsten Dynamiken? (Fukuyama 1992) Hierüber wird man in den nächsten Jahren und Jahrzenten sicher trefflich in und zwischen den verschiedenen Disziplinen streiten können; aus zeithistorischer Perspektive scheint vor allzu starken Qualifizierungen oder superlativischen Dramatisierungen noch etwas abwartende Zurückhaltung angebracht. So warnten Ökonomen und Politiker angesichts der derzeitigen einschneidenden Maßnahmen im Zuge der Covid19-Pandemie schon im Frühjahr vor der ,größten Krise“ wahlweise seit 1945, 1929, 1919, des letzten Jahrhunderts oder gar der gesamten „Menschheitsgeschichte“. Die langfristigen strukturellen Konsequenzen wie mentalen Folgewirkungen sind indes nur schwer absehbar, da sich Deglobalisierungprozesse und Digitalisierungsdynamiken eigentümlich $\mathrm{zu}$ vermischen und insbesondere die schon so oft verabschiedeten Nationalstaaten als gestaltungsmächtige Akteure mit ihrer Regulierungs- und Interventions-Macht eindrucksvoll auf die Weltbühne zurückgekehrt scheinen.

Ein heimisches Opfer der Corona-Entwicklungen steht jedoch bereits fest - das Gedenkjahr 2020. Mit dem abrupten Ausfall zahlreicher Konferenzen, Podiumsdiskussionen und Festveranstaltungen ist - zumindest vorerst - eine intensive Diskussion unvermittelt abgebrochen, die seit 2015 zunehmend an Fahrt gewonnen hatte. Hatten in den Jahrzehnten zuvor eine offiziöse (westdeutsche) Heldengeschichte vom normalisierenden „Erfolg“ von Revolution, Einigung und „Aufbau Ost“ mit einer

Dr. M. Böick (ه)

Professur für Zeitgeschichte/Historisches Institut, Ruhr-Universität Bochum, Bochum, Deutschland E-Mail: Marcus.Boeick@rub.de 
untergründigen (ostdeutschen) Opfergeschichte einer „Kolonialisierung“ mehr oder weniger unverbundenen nebeneinander ihr geschichtspolitisches Dasein gefristet, haben sich die Diskussionen in den letzten Jahren erheblich pluralisiert und dynamisiert. Diese oft recht nabelschauartigen Re-Bilanzierungsversuche in Deutschland sind auf ein komplexes Bündel an politischen, gesellschaftlichen wie generationellen Ursachen zurückzuführen: die wachsende zeitliche Distanz, die Erfolge der AfD gerade im Osten, der allmähliche Abschied der deutungsmächtigen Zeitzeugen und Geschichtspolitiker, die veränderten Blickwinkel jüngerer (Forscher/innen-)Generationen mit Biografien jenseits vermeintlich eindeutiger Ost-West-Dichotomien sowie europäische oder globale Perspektivsetzungen dienen als zentrale Impulse. In jedem Falle erschienen öffentliche Erinnerungskultur, politische Diskussion und auch die wissenschaftliche Debatte zum Komplex „1989/90“ erheblich in Bewegung geraten zu sein, als im Frühjahr 2020 auch hier eine unvermittelte Schockstarre und -stille eintrat (Mau 2019; Kowalczuk 2019; Hensel und Engler 2018).

\section{Die Treuhandanstalt - ein skandalumwittertes Gebilde, das in keine Schublade passt $(\mathrm{e})$}

Von diesem erinnerungskulturellen Lockdown betroffen war auch eine eigentümliche Schwellen-Organisation, die in den letzten Jahren symbolhaft erneut ins Zentrum dieser eigentümlich verkeilt-verkrusteten Ost-West-Aufrechnungen gerückt ist: die skandalumwitterte Treuhandanstalt. Diese hatte - beginnend vor allem mit der Wirtschafts- und Währungsunion im Juli 1990 - die krisenhaften Übergänge von der Plan- zur Marktwirtschaft in der ostdeutschen Betriebslandschaft wesentlich (aber sicher nicht exklusiv) mitgestaltet. (Steiner 2007) Für viele Ostdeutsche avancierten die Treuhand und ihr rasch rekrutiertes westdeutsches Führungspersonal nach 1991 zum herausragenden Symbol einer ,feindlichen Übernahme“ des Ostens durch westdeutsche Konzern- und Interessenvertreter, die statt der versprochenen ,blühenden Landschaften“ nun vielmehr in Kolonialherrenmanier Abwicklung, Arbeitslosigkeit und Abwanderung zu bringen schien. Nach intensiven Demonstrationen, Streiks und Protesten im gesamten „Beitrittsgebiet“ war es der tödliche Anschlag auf den Treuhand-Präsidenten Detlev Karsten Rohwedder am 1. April 1991, der als tiefer Einschnitt wahrgenommen wurde; die kurzen Flitterwochen nach der unverhofft-unvorbereiteten Einigung schienen endgültig vorbei; vielmehr stand nun der krisenhafte Ehealltag zweier Partner auf dem Programm, die sich erst aus der Nahperspektive ihrer erheblichen Differenzen mittlerweile nur allzu bewusst wurden (Rödder 2009).

Die umkämpfte und exponierte Treuhand rückte dabei schnell ins Zentrum einer veritablen „,Vereinigungskrise“ (Kocka 1995): Als „größtes Unternehmen der Welt" betreute diese ab Oktober 1990 dem Bundesfinanzministerium in Bonn unterstellte Behörde über achttausend Betriebe mit vier Millionen Beschäftigten, von denen sie bis Ende 1992 - also in nicht einmal zwei Jahren - knapp $80 \%$ im Eiltempo privatisiert oder abgewickelt hatte. Der Löwenanteil der Betriebe ging dabei an westdeutsche Investoren und Unternehmen, während Übernahmen durch ostdeutsche oder ausländische Kandidaten erheblich seltener auftraten. Jahrzehnte dirigistischer Zentralplanwirtschaft mit ihren massiven ökonomischen, ökologischen 
wie technologischen Konsequenzen und Fehlentwicklungen, der unvermittelte globale (Markt-)Schock der Wirtschafts- und Währungsunion sowie der dramatische Einbruch der Ostmärkte nach 1991 zollten bei der von der Treuhand forcierten Privatisierungspraxis einen enormen Tribut. Der Umbau der oft in enorm schwieriger Lage operierenden Ost-Betriebe war mit umfassenden Massenentlassungen, hitzigen industriepolitischen Diskussionen sowie massiven sozialen Protesten verbunden; viele hiervon Betroffene erlebten das abrupte Ende der spät-industriellen Lebens- und Arbeitswelten, die das SED-Regime zuvor wie in einer Seifenblase erhalten hatte, als enormen biografischen Einschnitt (Böick 2018).

Die Treuhand und ihr widersprüchliches Wirken lagen damit letztlich quer zu allen altbundesrepublikanisch-bekannten Schubladen von Politik und Wirtschaft, Staat und Markt, Ost und West, Zentrum und Peripherie: Sie war als bundesunmittelbare Agentur formal eine staatliche Behörde, agierte aber in ihrem kulturellen Selbstverständnis sowie ihren operativen Strategien und organisationellen Strukturen oft wie ein marktorientiertes Unternehmen; sie gestaltete die östliche Planwirtschaft mit westdeutschem Expertenpersonal in eine westliche Marktordnung um und verquickte dabei strukturell-volkswirtschaftliche mit unternehmerisch-betriebswirtschaftlichen Aufgaben; sie handelte in einer Art auf Dauer gestellten Ausnahmezustand bisweilen hochgradig zentralistisch, hatte sich aber zunehmend mit einem sich verdichtenden föderalen wie regionalen Geflecht an Akteuren und Veto-Spielern (Verbänden, Gewerkschaften, Landesregierungen, Kommunen) zu arrangieren. Die Treuhand avancierte dabei de facto zu einer Art Wirtschaftsregierung im Osten und erschien bereits den zeitgenössischen Beobachtern, wie noch zu zeigen sein wird, als bemerkenswert-exzeptionelle Anomalie im politischen System der erweiterten Bundesrepublik (Czada 1993; Tsebelis 2002).

\section{Kurzfristige Annäherungen an die Transformation und das „Monster“ im Osten}

Es verwundert kaum, dass der verworren-skandalumwitterte Treuhand-Komplex bald auch in der gegenwartsorientierten Wissenschaftslandschaft erhebliches Aufsehen erregte. 1990 erschien die Stimmungslage in den bundesdeutschen Sozialwissenschaften eigentümlich zweigeteilt: Einerseits steckte vielen Experten der „Prognoseschock" über die auch von etablierten DDR-Forschern nicht vorhergesehene Implosion des sowjetischen Machtbereichs spürbar in den Knochen. Andererseits griff rasch eine regelrechte Euphorie über das beginnende soziale „Großexperiment“ um sich, bei dem man wie in einer Art sozialen Labor im „Zeitraffer“ gesellschaftliche Wandlungsdynamiken begleitend mitverfolgen könne, wie Claus Leggewie und Reinhard Giesen bereits 1991 in merklicher Aufbruchsstimmung formulierten. (Giesen und Leggewie 1991) Diese frühe Transformationsforschung konnte zunächst erhebliche Ressourcen und Aufmerksamkeit akquirieren, litt aber schon bald unter einem abflauenden öffentlichen Interesse und hatte selbst - quasi als Teil dieses Prozesses - mit internen Ost-West-Spannungen zu kämpfen: Der massive Umbau der ostdeutschen Hochschullandschaft erschien als zentrales Aktionsfeld, der nicht zuletzt viele westdeutsche Akademiker an oft neugründeten Instituten führte, während 
die zumeist politisch diskreditierten ostdeutschen Forscher ihrer Abwicklung oder anderweitigen Arbeitsbeschaffungsmaßnahmen entgegensahen (Kollmorgen et al. 2014).

Die Politikwissenschaft interessierte sich zunächst bevorzugt für die zuvor kaum vorstellbaren außen- wie innenpolitischen Ereignisse und Dynamiken von Revolution und Einigung der Jahre 1989/90 im engeren Sinne, die Klaus von Beyme markant als „Stunde der Exekutive“ charakterisiert hatte. (von Beyme 2004; Grosser 1998) Aber auch jenseits dessen lagen spannende Themen für die Politikwissenschaftler/ innen buchstäblich auf der Straße, wie das von Werner Weidenfeld und Karl-Rudolf Korte bereits 1992 erstmals herausgegebene „Handbuch zur deutschen Einheit“ eindrucksvoll demonstrierte. (Weidenfeld und Korte 1999) An hochaktuellen Untersuchungsgegenständen herrschte kein Mangel - seien es der Neuaufbau der Länder, die außenpolitisch kniffligen Grenz-, Bündnis- oder Europafragen, die intensiven Verfassungs- und Hauptstadtdiskussionen oder die geschichtspolitischen Kontroversen über das Erbe von „Stasi“, SED sowie Nationalsozialismus und Holocaust. Unter dem drängenden Eindruck rechtsextremer Pogrome und grassierender Fremdenfeindlichkeit scheinen insbesondere auch mentalitätsbezogene Fragen nach einer anders gearteten, ,post-sozialistischen“ politischen Kultur und überaus kontrovers diskutierten „Ost-Identitäten“ vordringlich, die neben der SED-Nachfolgepartei PDS auch merklich andere Wahlergebnisse und in der alten Bundesrepublik unbekannte Regierungskonstellationen hervorbrachten (Esser 2000; Thumfart 2002).

Der Treuhandanstalt kam in dieser oft noch stark dokumentarisch-chronistisch ausgerichteten Forschungskonjunktur eine eigentümliche Sonderrolle zu, war sie doch selbst ein ausgesprochen zwitterhaftes Gebilde, dessen schwer durchschaubare Arbeitsfelder letztlich quer zu etablierten Disziplingrenzen lag - neben den Politikwissenschaftlern beanspruchten Ökonomen und Juristen Deutungshoheiten, taten sich aber zugleich mit übergreifenden Synthesen schwer. Gerade in ihrer heißen Frühphase, in den Jahren 1991/92, als die Treuhand bis zu fünfhundert Privatisierungen pro Monat vermeldete und die damit verknüpften Debatten und Proteste enorm an Fahrt aufgenommen hatten, schien es für die zeitgenössischen Forscher/ innen kaum möglich, sich selbst ein empirisch fundiertes Bild oder gar eine Stimme in den hitzigen Konflikten zu verschaffen. Das in den Transformationskonflikten umkämpfte „Monster“ Treuhand schien mit den differenzierenden Mitteln und Methoden der Wissenschaft kaum sinnvoll zu bändigen - bis diese aus eigenem Antrieb eine veritable Zahl an Forschern Ende 1992 zu einem umfangreichen Forschungsprojekt in die geschichtsträchtige „Festung“ im einstigen Reichsluftfahrtministerium (und Gründungsort der DDR), die seit März 1991 der Treuhand-Zentrale als Sitz diente, einlud (Kemmler 1994).

\section{Mittelfristige Reflexionen zum Ort einer exzeptionellen „Nebenregierung Ost“"}

Es war also die hochumstrittene Organisation selbst, die eine erste Forschungswelle aktiv befeuerte. Unter der Führung der beiden prominenten Ökonomen Hans Karl Schneider und Herbert Hax (immerhin der amtierende Vorsitzende der ,Wirt- 
schaftsweisen“" und sein Amtsvorgänger) sowie dem Wirtschaftshistoriker Wolfram Fischer sollten zwei Dutzend Wissenschaftler hinter die Treuhand-Kulissen blicken dürften. Den eilig beauftragten Forschern bot man exklusive Recherchemöglichkeiten, Zugang zu Führungspersonal oder entsprechende Akteneinsichtnahmen, wobei das Resultat bereits ein Jahr später in einem dicken Sammelband unter dem sprechenden Namen „Treuhandanstalt - das Unmögliche wagen“ publiziert wurde. Die Forscher - nahezu sämtlich westdeutsche Männer - gelangten zu einem differenzierten Ergebnis; die Treuhand schien letztlich, eingedenk der zahlreichen Probleme und Widersprüche, besser zu sein als ihr hundsmiserabler Ruf. Ein Befund, der in der aufgepeitschten Medienöffentlichkeit zumeist Häme und Misstrauen gegenüber der bezahlten „Auftragsarbeit“ aus einem fernen „Elfenbeinturm“ auslösen sollte (Fischer et al. 1993).

Während mehrheitlich Juristen und Ökonomen einen Großteil der in großer Eile zusammengetragenen Beiträge beisteuerten, waren mit Wolfgang Seibel und Roland Czada auch zwei jüngere Politik- bzw. Verwaltungswissenschaftler aus Konstanz mit je eigenen Forschungsbeiträgen vertreten, die sich durchaus von den zumeist affirmativ-kleinteilig gehaltenen ökonomischen wie juristischen Fachbeiträgen unterschieden. Wolfgang Seibel richtete seine verwaltungswissenschaftliche Analyse des kaum bekannten „Innenlebens“ der Treuhand und ihres Personals auf die verschiedenen historischen Entwicklungsphasen sowie die hiermit verbundenen organisationskulturellen Eigendynamiken aus. Das sozial überaus heterogen zusammengesetzte Treuhand-Personal erschien ihm - aller unbestreitbaren sozialen wie kulturellen Differenzen zwischen „Beamten“ und „Managern“, „Ossis“ und „Wessis“, „Männern“ und „Frauen“ sowie „Alten“ und ,Jungen“ zum Trotz - durch eine spezifische „Treuhand-Kultur“ zusammengehalten, die sich insbesondere durch die äußeren Widerstände sowie den ,lebendigen Prozeß der Zielerreichung“ einer im Grunde kaum bearbeitbaren Aufgaben- und Problemfülle im Innersten dieses Sonder-Gebildes beinahe wagenburgartig herausgebildet hatte. (Seibel 1993, S. 143; Weber 1996).

Während sich Seibel für das dynamische Innenleben interessierte, thematisierte sein Kollege Roland Czada die äußeren Kontexte und externen Beziehungsmuster. Er zielte darauf ab, die Organisation ,,im Umfeld von Politik und Verbänden“ zu verorten und diskutierte dabei ihre eigentümliche Zwischenstellung zwischen Bund, Ländern und Kommunen; auch beschrieb er ihre Sonderrolle im Kontext der politisch-korporativen Netzwerke, die sich um den „Aufbau Ost“ zu ranken begannen und die auch Gewerkschaften und Verbände miteinschlossen. Als unverhoffte „Nebenregierung-Ost" deutete Czada die Treuhand letztlich als Ausdruck der Tatsache, dass die von den Umbrüchen überraschte konservativ-liberale Bundesregierung im Jahr 1990 allzu naiv auf die ,Selbstheilungskräfte des Marktes“ vertraut und die ,politisch-institutionellen Voraussetzungen“ demgegenüber merklich unterschätzt hätte. Da aber kompetitive Mechanismen des „Markts“ oder die staatliche „Hierarchie“ allein den krisenhaften Wirtschaftsumbau kaum zu steuern in der Lage seien, bleibe nur „,das politische Kompromißprinzip verflochtener Entscheidungsstrukturen“ übrig. Am Ende, so Czada in seinem ernüchternden Fazit, habe die Treuhandanstalt von ,allen Aufgaben“, die man ihr seit 1990 hastig aufgebürdet habe, ,diese eine 
wirklich gemeistert: Regierungen, Interessengruppen und die gesamte Öffentlichkeit zu desillusionieren“ (Czada 1993, S. 171 f.; Czada 1994).

Die frühen Erkundungsarbeiten Czadas und Seibels markieren damit wichtige zeitgenössische politikwissenschaftliche Analyse- und Diagnose-Versuche zum noch schwer greifbaren „Treuhand-Komplex“, den sie auch in weiteren Studien weiter verfolgen sollten - im Gegensatz zu einem Großteil ihrer Mitstreiter im „Forschungsprojekt", die rasch das Interesse am Gegenstand verloren. Aus politikwissenschaftlicher Perspektive erschien die Organisation wie eine aus dem ,zentralistischen Erbe“ des SED-Regimes in die Gegenwart hineinragende Anomalie, eben ein regelrechtes „Ausnahmeregime“ im politischen System, das diesem aber zugleich - wie Wolfgang Seibel prägnant ausführte - als institutioneller „Blitzableiter“ in der Krise diente: Die enttäuschten Hoffnungen bzw. in Ostdeutschland gehegten „Illusionen“ über den Wert des einstigen Volksvermögens, entluden sich nun genau an dieser Stelle. Der unmittelbare Frust vieler Ostdeutscher über Schließungen und Entlassungen richtete sich gerade nicht gegen die eigentlich zuständige Bundesregierung bzw. das politische System als solches, sondern gegen die im Vordergrund agierende Treuhand. So funktional diese Schutzschildwirkung in den Diagnosen der frühen 1990er-Jahre einerseits erschien, desto weniger war aber auch Seibel und Czada zugleich die schwer durchschaubare und demokratisch schwach legitimierte Macht- und Aufgabenfülle der Organisation letztlich geheuer - sie erschien alles in allem als ausgesprochener Fremdkörper in einem seit Jahrzehnten eingespielten föderal-korporatistischen Verbund-System an Verflechtungen, der auf Dauer keine Existenzberichtung haben würde (Seibel 1994, 2005; Czada 2012).

\section{Langfristige Spuren eines erinnerungskulturellen „Mythos“}

Derlei Analyseversuche aus den frühen 1990er-Jahren waren bereits von der Frage bestimmt, was eigentlich langfristig von der Treuhand überdauern würde. Tatsächlich hatte diese bereits Ende 1992 ihre zehntausendste Privatisierung verkündet und sah sich auf einen guten Weg zu ihrer finale „Selbstauflösung“, die die Organisationsspitze um die Rohwedder-Nachfolgerin und einstige niedersächsische CDU-Ministerin Birgit Breuel propagierte und mit Nachdruck vorantrieb. Doch statt in Ruhe ihrem Ende entgegenzugehen, versank die Organisation gerade in den Jahren 1993 und 1994 in einem kaum enden wollenden Reigen an medialen Enthüllungen über Korruptionsfälle, spektakulären Protesten (etwa in Bischofferode im Sommer 1993) sowie politischen Diskussionen, die einen letzten Höhepunkt in einem ab Herbst 1993 tagenden Bundestagsuntersuchungsausschuss unter der Leitung von Otto Schily erlebten, der das Thema erstmals auch breit wie kontrovers in den parlamentarischen Raum hineintragen sollte. Als die Treuhand am 31. Dezember 1994 offiziell ihre Pforten schloss, übernahm die neue Bundesanstalt für vereinigungsbedingte Sonderaufgaben (BvS) zwar einen Großteil ihrer Aufgaben und ihres Personals, sollte aber nicht einmal ansatzweise die Aufmerksamkeit ihrer hochumstrittenen Vorgängerin erreichen (Bundesanstalt für vereinigungsbedingte Sonderaufgaben 2003).

Während im medienöffentlichen Raum vor allem anlässlich allfälliger Jahrestage die Skandale, Proteste und Debatten um den finsteren ,Treuhand-Komplex“ 
weiter nachhallten, ließ mit der Zeit auch das sozial- und politikwissenschaftliche Interesse an der Transformationszeit im Allgemeinen wie der Treuhandanstalt im Besonderen merklich nach. Sieht man von einer Reihe von Spezialstudien sowie weiteren Arbeiten von Czada und Seibel ab, rückten die Treuhandanstalt und der Wirtschaftsumbau spätestens ab dem Jahr 2000 weitgehend aus dem politikwissenschaftlichen Fokus. Dieses mittlerweile verschwundene Gebilde erschien nun, wie Stephan Weingarz in seiner ernüchternden Zwischenbilanz zur deutschen Transformationsforschung festhielt, als zu spezieller wie historischer Einzel-, Grenz- oder Extremfall, als dass dieses sich für weiterführende Theorie- und Prognosebildungen anbot; zugleich erschwerte ein vollständig unzugängliches Treuhand-Archiv in den ersten anderthalb Jahrzehnten des neuen Jahrtausends weiterführende empirische bzw. historische Arbeiten (Weingarz 2003; Best und Holtmann 2012).

Dies änderte sich allmählich erst ab Mitte der 2010er-Jahre, als die im Grunde weitgehend vergessene Treuhand erneut zum Thema in Politik, Öffentlichkeit und Wissenschaft wurde. Dabei war diese gar nicht wirklich ,verschwunden“, wie Böick und Goschler in einer explorativen Studie zum Stellenwert zur Wahrnehmung und Bewertung der Treuhandanstalt in der Erinnerungskultur in den Jahren 2016/17 zeigen konnten: Gerade Ostdeutschen in den mittleren bzw. älteren Altersgruppen über vierzig Jahren erschien insbesondere die Treuhand als außerordentlich negativ besetztes Symbol einer rigorosen „Abwicklung“ des Ostens durch den Westen unheimlich präsent, während sie insbesondere jüngeren Westdeutschen so gut wie unbekannt war. Die erinnerungskulturellen Gräben verliefen nicht nur zwischen Ost und West, sondern gerade auch zwischen den verschiedenen Generationen, was im Fazit der Kurzstudie auf die Formel einer „Bad Bank“ gebracht worden ist: Für die einstmals mittel- oder unmittelbar von den massiven Umbrüchen nach 1990 individuell Betroffenen hatte sich ,die“ Treuhand - von der Öffentlichkeit weitgehend unbemerkt - zu einem wirkmächtigen Negativ-Mythos verdichtet, der nun symbolisch für die erlittenen Enttäuschungs- und Deklassierungserfahrungen stand, die viele Ostdeutsche in den 1990er-Jahren mit dem bundesdeutschen Modell von liberaler Demokratie und kapitalistischer Marktwirtschaft gesammelt hatten (Böick und Goschler 2017).

\section{Fazit: neue Brückenschläge zwischen Politikwissenschaft und Zeitgeschichte?}

Aus dem funktionalen „Blitzableiter“ war damit, wie man pointieren könnte, eine in ihren langfristigen mentalen Wirkungen durchaus dysfunktionale „Bad Bank“ geworden, die in ihren gegenkulturellen Mystifizierungen auch leicht politisier- und polarisierbar erscheint. So war es im Grunde kaum verwunderlich, dass in den ostdeutschen Landtagswahlkämpfen des Jahres 2019 neben der traditionell Treuhand-kritischen Linken auch die rechtspopulistische AfD das Thema mit Nachdruck aufgriff und sich medienwirksam den linken Forderungen nach einem neuerlichen Untersuchungsausschuss im Bundestag anschloss. Der negative Mythos „Treuhand“ rückte damit wieder in den Fokus der Öffentlichkeit, die anhand dieser Geschichte die noch immer merklichen materiellen wie mentalen Nachwirkungen von DDR 
und Transformationszeit thematisierte - in zahlreichen neuen TV-Dokumentationen, Ausstellungs- und Theaterprojekten sowie populären und bisweilen hochkontrovers diskutierten politischen oder journalistischen Publikationen (Köpping 2018; Pötzl 2019).

Doch auch die Wissenschaft hat das lange randständige Themenfeld mittlerweile neu für sich entdeckt. Eine Reihe von größeren ökonomischen und nun auch geschichtswissenschaftlichen Forschungsprojekten hat damit begonnen, die sukzessive durch das Bundesarchiv erschlossenen und freigegebenen Quellen- und Datenbestände für detaillierte Analysen und empirische Fallstudien zu nutzen. In diesem Zusammenhang knüpfen die Wissenschaftler/innen freilich auch an die Studien und Resultate der ersten Generation von Treuhand-Forscher/innen an. Es wird dabei eine besondere Herausforderung sein, die umfassenden Befunde, Deutungen und (Roh-)Daten der ersten Wellen der zeitgenössischen Transformationsforschung produktiv zu historisieren (Hoffmann 2020).

Gerade für Historiker/innen erweisen sich von Sozial- und Politikwissenschaftler/innen durchgeführte, zeitgenössische Interviewreihen, erhobene Befragungsdaten oder detaillierte Netzwerkbeschreibungen als komplexe, aber ungemein lohnenswerte Sammlung an wissenschaftlich produzierten Quellengruppen, über deren Stellenwert in der Zeitgeschichtsforschung selbst seit einigen Jahren methodisch intensiv diskutiert wird. In beiden Fächern könnte aber die Offenheit und Bereitschaft für konzeptionellen, theoretischen wie empirischen Austausch in der Praxis deutlich stärker ausfallen (Graf und Priemel 2011).

Aber auch interdisziplinär könnte eine neuerliche Welle einer räumlich, thematisch wie methodisch deutlich erweiterten Transformationsforschung - die eben gerade nicht nur regionale „Ostdeutschlandforschung“ sein sollte - erhebliche Diskussions- und Kooperationspotenziale zwischen zwei traditionell sehr eng verwandten Fächern bieten, die sich in den letzten Jahrzehnten allerdings merklich voneinander entfernt haben - eine immer stärker quantitativ-theoretisch orientierte Politikwissenschaft hier, eine demgegenüber vorwiegend qualitativ-empirisch arbeitende Zeitgeschichtswissenschaft dort.

Wie sich aber gerade am Extrem-Beispiel der Treuhand und ihrer überaus bewegten Entstehungs- und Erinnerungsgeschichte vom „Blitzableiter“ zur „Bad-Bank“ exemplarisch beobachten ließ, wären vor allem die Temporalitäten und Transformationen von politischen wie gesellschaftlichen Ordnungsmustern in ihrer intensiven Verschränkung mit zeitgenössischen Krisen- und Transformationswahrnehmungen ein hochinteressantes Schnittfeld, das beide Fächer wieder stärker in eine gemeinsame Diskussionen hineinführen könnte: So hatte der Wiener Historiker Philipp Ther vor einiger Zeit eine Debatte über mögliche „Ko-Transformationen“ angeregt, bei denen die osteuropäischen Umbrüche mittelbar auch auf Westeuropa zurückgewirkt hätten - eine Diskussion, die kürzlich auch Ivan Krastev und Stephen Holmes prominent mit einer düsteren Enttäuschungsdiagnose fortgesetzt haben. Aber auch jenseits Europas stellte der kanadische Historiker Quinn Slobodian eine globalgeschichtliche Historisierung eines staatsskeptischen Neoliberalismus als globaler Ideen- und Herrschaftsform zur Diskussion, die natürlich auch Kernfragen der Politikwissenschaft berührt. All diese Fragen könnten als Ausgangspunkte für neue Diskussions- 
und Verbindungslinien zwischen den Fächern dienen - ob in digitaler oder analoger Form (Ther 2014; Krastev und Holmes 2019; Slobodian 2018).

Funding Open Access funding provided by Projekt DEAL.

Open Access Dieser Artikel wird unter der Creative Commons Namensnennung 4.0 International Lizenz veröffentlicht, welche die Nutzung, Vervielfältigung, Bearbeitung, Verbreitung und Wiedergabe in jeglichem Medium und Format erlaubt, sofern Sie den/die ursprünglichen Autor(en) und die Quelle ordnungsgemäß nennen, einen Link zur Creative Commons Lizenz beifügen und angeben, ob Änderungen vorgenommen wurden.

Die in diesem Artikel enthaltenen Bilder und sonstiges Drittmaterial unterliegen ebenfalls der genannten Creative Commons Lizenz, sofern sich aus der Abbildungslegende nichts anderes ergibt. Sofern das betreffende Material nicht unter der genannten Creative Commons Lizenz steht und die betreffende Handlung nicht nach gesetzlichen Vorschriften erlaubt ist, ist für die oben aufgeführten Weiterverwendungen des Materials die Einwilligung des jeweiligen Rechteinhabers einzuholen.

Weitere Details zur Lizenz entnehmen Sie bitte der Lizenzinformation auf http://creativecommons.org/ licenses/by/4.0/deed.de.

\section{Literatur}

Best, Heinrich, und Everhard Holtmann (Hrsg.). 2012. Aufbruch der entsicherten Gesellschaft: Deutschland nach der Wiedervereinigung. Frankfurt a.M.: Campus.

von Beyme, Klaus. 2004. Das politische System der Bundesrepublik Deutschland: Eine Einführung. Wiesbaden: Springer.

Böick, Marcus. 2018. Die Treuhand: Idee - Praxis. Göttingen: Wallstein.

Böick, Marcus, und Constantin Goschler. 2017. Wahrnehmung und Bewertung der Arbeit der Treuhandanstalt. Bochum: Bundesministerium für Wirtschaft und Energie.

Bundesanstalt für vereinigungsbedingte Sonderaufgaben (Hrsg.). 2003. Schnell privatisieren: entschlossen sanieren, behutsam stilllegen. Ein Rückblick: auf 13 Jahre Arbeit der Treuhandanstalt und der Bundesanstalt für Vereinigungsbedingte Sonderaufgaben. Berlin: Wegweiser.

Czada, Roland. 1993. Die Treuhandanstalt im Umfeld von Politik und Verbänden. In Treuhandanstalt: Das Unmögliche wage, Hrsg. Wolfram Fischer, Herbert Hax, und Hans-Karl Schneider, 148-173. Berlin: Akademie.

Czada, Roland. 1994. Die Treuhandanstalt im politischen System der Bundesrepublik. Aus Politik und Zeitgeschichte 43/44:31-42.

Czada, Roland. 2012. Das Erbe der Treuhandanstalt. In Einheit - Eigentum - Effizienz: Bilanz der Treuhandanstalt, Hrsg. O. Depenheuer, K.-H. Paqué, 125-146. Berlin, Heidelberg, New York: Springer.

Esser, Hartmut (Hrsg.). 2000. Der Wandel nach der Wende. Gesellschaft, Wirtschaft, Politik in Ostdeutschland. Wiesbaden: Westdeutscher Verlag.

Fischer, Wolfram, Herbert Hax, und Hans-Karl Schneider (Hrsg.). 1993. Treuhandanstalt: Das Unmögliche wagen. Berlin: Akademie.

Fukuyama, Francis. 1992. The end of history and the last man. London: Penguin Books.

Giesen, Bernd, und Claus Leggewie (Hrsg.). 1991. Experiment Vereinigung: Ein sozialer Großversuch. Berlin: Rotbuch.

Grosser, Dieter. 1998. Das Wagnis der Währungs-, Wirtschafts- und Sozialunion. Politische Zwänge im Konflikt mit ökonomischen Regeln. Stuttgart: Deutsche Verlags-Anstalt.

Hensel, Jana, und Wolfgang Engler. 2018. Wer wir sind: Die Erfahrung, ostdeutsch zu sein. Berlin: Aufbau.

Hoffmann, Dierk (Hrsg.). 2020. Transformation einer Volkswirtschaft. Neue Forschungen zur Geschichte der Treuhandanstalt. Berlin: Metropol.

Kemmler, Marc. 1994. Die Entstehung der Treuhandanstalt: Von der Wahrung zur Privatisierung des DDR-Volkseigentums. Frankfurt a.M.: Campus.

Kocka, Jürgen. 1995. Vereinigungskrise. Zur Geschichte der Gegenwart. Göttingen: Vandenhoeck \& Ruprecht.

Kollmorgen, Raj, Wolfgang Merkel, und Hans-Jürgen Wagener (Hrsg.). 2014. Handbuch Transformationsforschung. Wiesbaden: Springer. 
Köpping, Petra. 2018. „Integriert doch erst mal uns!“ Eine Streitschrift für den Osten. Berlin: Christoph Links.

Kowalczuk, Ilko-Sascha. 2019. Die Übernahme. Wie Ostdeutschland Teil der Bundesrepublik wurde. München: C.H. Beck.

Krastev, Ivan, und Stephen Holmes. 2019. The light that failed. A reckoning. London: Allen Lane.

Mau, Steffen. 2019. Lütten Klein. Leben in der ostdeutschen Transformationsgesellschaft. Berlin: Suhrkamp.

Pötzl, Norbert F. 2019. Der Treuhand-Komplex. Legenden. Fakten. Emotionen. Hamburg: Kursbuch.

Rödder, Andreas. 2009. Deutschland einig Vaterland: Die Geschichte der Wiedervereinigung. München: C.H. Beck.

Rüdiger, Graf, und Kim Christian Priemel. 2011. Zeitgeschichte in der Welt der Sozialwissenschaften. Legitimität und Originalität einer Disziplin. Vierteljahrshefte für Zeitgeschichte 59(4):1-30.

Seibel, Wolfgang. 1993. Die organisatorische Entwicklung der Treuhandanstalt. In Treuhandanstalt: Das Unmögliche wagen, Hrsg. Wolfram Fischer, Herbert Hax, und Hans-Karl Schneider, 111-147. Berlin: Akademie.

Seibel, Wolfgang. 1994. Das zentralistische Erbe: Die institutionelle Entwicklung der Treuhandanstalt und die Nachhaltigkeit ihrer Auswirkungen auf die bundesstaatlichen Verfassungsstrukturen. Aus Politik und Zeitgeschichte 43/44:3-13.

Seibel, Wolfgang. 2005. Verwaltete Illusionen: Die Privatisierung der DDR-Wirtschaft durch die Treuhandanstalt und ihre Nachfolger 1990-2000. Frankfurt a.M.: Campus.

Slobodian, Quinn. 2018. Globalists. The end of empire and the birth of neoliberalism. Cambridge: Harvard University Press.

Steiner, André. 2007. Von Plan zu Plan: Eine Wirtschaftsgeschichte der DDR. Berlin: Aufbau Taschenbuch.

Ther, Philipp. 2014. Die neue Ordnung auf dem alten Kontinent: Eine Geschichte des neoliberalen Europa. Berlin: Suhrkamp.

Thumfart, Alexander. 2002. Die politische Integration Ostdeutschlands. Frankfurt a.M.: Suhrkamp.

Tsebelis, George. 2002. Veto players. How political institutions work. Princeton: Princeton University Press.

Weber, Christiana. 1996. Treuhandanstalt. Eine Organisationskultur entsteht im Zeitraffer. Wiesbaden: Westdeutscher Verlag.

Weidenfeld, Werner, und Karl-Rudolf Korte (Hrsg.). 1999. Handbuch zur deutschen Einheit. 1949-1989-1999. Frankfurt a.M.: Campus.

Weingarz, Stephan. 2003. Laboratorium Deutschland? Der ostdeutsche Transformationsprozess als Herausforderung für die deutschen Sozialwissenschaften. München: Lit. 